\title{
An alternative phase-space distribution to sample initial conditions for classical dynamics simulations
}

\author{
A. García-Vela \\ Instituto de Matemáticas y Física Fundamental, \\ C.S.I.C., Serrano 123, 28006 Madrid, Spain
}

\begin{abstract}
A new quantum-type phase-space distribution is proposed in order to sample initial conditions for classical trajectory simulations. The phase-space distribution is obtained as the modulus of a quantum phase-space state of the system, defined as the direct product of the coordinate and momentum representations of the quantum initial state. The distribution is tested by sampling initial conditions which reproduce the initial state of the $\mathrm{Ar}-\mathrm{HCl}$ cluster prepared by ultraviolet excitation, and by simulating the photodissociation dynamics by classical trajectories. The results are compared with those of a wave packet calculation, and with a classical simulation using an initial phase-space distribution recently suggested. A better agreement is found between the classical and the quantum predictions with the present phase-space distribution, as compared with the previous one. This improvement is attributed to the fact that the phase-space distribution propagated classically in this work resembles more closely the shape of the wave packet propagated quantum mechanically.
\end{abstract}

Keywords: Phase-space distribution; Classical dynamics; Ultraviolet photolysis E-mail address: garciavela@imaff.cfmac.csic.es

Phone: 34-915616800-1103

Fax: 34-915854894 


\section{Introduction}

The classical Molecular Dynamics (MD) technique has been extensively applied to a wide variety of dynamical problems [1-19], due to its efficiency and simplicity of implementation. One of the features that differenciates the MD methodology from the quantum mechanical one is the representation of the state of the system at any time. In classical mechanics the system must be represented in phase space (PS), i.e. both in coordinate and in momentum space simultaneously, while in quantum mechanics only either the coordinate or the momentum representation is required. Correspondingly, in the classical framework the initial state of the system must be specified in phase space. Thus, in order to apply a classical dynamics method, if the system initial state calculated quantum mechanically, usually represented in coordinate space, is to be used, it has to be transformed into a PS representation. Such a transformation is not unique.

Several models have been proposed to generate a PS distribution of initial conditions for classical trajectories from the quantum initial state of the system in coordinate space. One of the PS distributions most extensively used was proposed by Wigner [20]. Other initial PS distributions have been suggested and applied [21-26]. An alternative to the above quantum-type PS distributions is to generate a purely classical distribution of initial conditions $[8,9,18]$. In this case the calculation of the quantum initial state in coordinate space, and its further transformation into a PS representation is unnecessary. However, such a classical distribution becomes unrealistic when the ground or a low-excited initial state of the system is involved.

In an earlier work [27] a new quantum-type PS distribution to sample initial conditions was proposed. This distribution is close in spirit to the Wigner distribution, although it is found to be more advantageous, mainly in terms of efficiency as the number of system modes increases. The suggested distribution is based on a definition of a quantum PS state of the system as the direct product of the coordinate and momentum representations of the quantum initial state. The PS distribution was defined as the square modulus of this PS state. The distribution was tested by 
carrying out a classical dynamics simulation of the $\mathrm{Ar}-\mathrm{HCl}$ ultraviolet photolysis, and comparing the results with those of an exact wave packet calculation. Good agreement was found in general between the classical and the quantum results. One of the conclusions of that work was that the agreement between the classical and the quantum predictions improves as the shape of the initial PS distribution approaches the shape of the initial wave packet which is propagated quantum mechanically.

The above conclusion has the interesting implication that a PS distribution defined as the modulus of the quantum PS state could provide a better agreement between the classical and the quantum results than the distribution previously suggested. In this paper such a possibility is investigated for the ultraviolet photolysis of the $\mathrm{Ar}-\mathrm{HCl}$ cluster.

The paper is organized as follows. In Section 2 the method to obtain the PS distribution and the sampling technique applied are described. The results of the classical simulation are compared with exact quantum ones and discussed in Section 3. Some conclusions are given in Section 4.

\section{Methodology}

\subsection{Initial state}

The calculation of the quantum initial state of the system is the first step in order to generate the PS distribution. The $\mathrm{Ar}-\mathrm{HCl}$ cluster is represented in Jacobian coordinates $(r, R, \theta)$, where $r$ is the $\mathrm{HCl}$ separation, $R$ is the distance between the Ar atom and the $\mathrm{HCl}$ centre of mass, and $\theta$ is the angle between the vectors $\mathbf{r}$ and $\mathbf{R}$. In the definition adopted for $\theta, \theta=0^{\circ}$ corresponds to the linear geometry $\mathrm{Ar}-\mathrm{H}-\mathrm{Cl}$. Zero total angular momentum is assumed for the system. The Hamiltonian takes the form

$$
\hat{H}(r, R, \theta)=-\frac{\hbar^{2}}{2 \mu_{r}} \frac{\partial^{2}}{\partial r^{2}}-\frac{\hbar^{2}}{2 \mu_{R}} \frac{\partial^{2}}{\partial R^{2}}+\left(\frac{1}{2 \mu_{r} r^{2}}+\frac{1}{2 \mu_{R} R^{2}}\right) \hat{j}^{2}+V_{H C l}(r)+W(r, R, \theta),
$$

being $\mu_{r}$ and $\mu_{R}$ the reduced masses associated with the $r$ and $R$ modes, respectively, and $W(r, R, \theta)$ is the interaction potential of $\mathrm{Ar}$ with $\mathrm{H}$ and $\mathrm{Cl}$. The potential surface 
where the initial state is calculated $[28,29]$ corresponds with the ground electronic state $X^{1} \Sigma^{+}$of $\mathrm{Ar}-\mathrm{HCl}$.

The initial state is defined as

$$
\Phi(r, R, \theta, t=0)=\chi_{v}(r) \varphi^{(v)}(R, \theta)
$$

where $\chi_{v}(r)$ is the ground vibrational state $(v=0)$ of $\mathrm{HCl}$, calculated as a numerical solution of the equation

$$
\left[-\frac{\hbar^{2}}{2 \mu_{r}} \frac{\partial^{2}}{\partial r^{2}}+V_{H C l}(r)\right] \chi_{v}(r)=E_{v} \chi_{v}(r),
$$

and $\varphi^{(v)}(R, \theta)$ is calculated variationally, and also corresponds with the ground rovibrational state of the van der Waals (vdW) modes. In order to simplify the calculation of the initial state, the $r$ dependence has been separated by applying the vibrational diabatic approximation [30]. Such a separation is physically justified in the ground electronic state of $\mathrm{Ar}-\mathrm{HCl}$, because of the large difference between the vibrational frequencies of the $\mathrm{HCl}$ stretch and the vdW modes. This separation, however, does not affect the definition of the corresponding PS distribution.

\subsection{Definition of the initial phase-space distribution function}

From the quantum initial state $\Phi(r, R, \theta)$ a quantum PS state of the system is defined as the direct product $\Phi(r, R, \theta) \tilde{\Phi}\left(p_{r}, p_{R}, j\right)[27]$, where $\tilde{\Phi}\left(p_{r}, p_{R}, j\right)$ is the momentum representation of $\Phi(r, R, \theta)$,

$$
\tilde{\Phi}\left(p_{r}, p_{R}, j\right)=\frac{1}{2 \pi \hbar} \int d r \int d R \int d \theta \sin \theta e^{-i p_{r} r / \hbar} e^{-i p_{R} R / \hbar} P_{j}(\cos \theta) \Phi(r, R, \theta),
$$

being $P_{j}(\cos \theta)$ a normalized Legendre polynomial. The above definition of the quantum PS state presents similarities with the PS distribution proposed by Kirkwood $[21,23]$. Now, our PS distribution is defined as

$$
P\left(r, R, \theta, p_{r}, p_{R}, j\right)=\left|\Phi(r, R, \theta) \tilde{\Phi}\left(p_{r}, p_{R}, j\right)\right|,
$$

or equivalently,

$$
P\left(r, R, \theta, p_{r}, p_{R}, j\right)=|\Phi(r, R, \theta)|\left|\tilde{\Phi}\left(p_{r}, p_{R}, j\right)\right|,
$$


since $\Phi(r, R, \theta)$ is a real function. In our present case where $\Phi(r, R, \theta)$ corresponds to the system ground state, and it is therefore positive everywhere, one can write

$$
P\left(r, R, \theta, p_{r}, p_{R}, j\right)=\Phi(r, R, \theta)\left|\tilde{\Phi}\left(p_{r}, p_{R}, j\right)\right|
$$

The relation $p_{\theta}=[j(j+1)]^{1 / 2} \hbar$ allows one to obtain the PS distribution, $P\left(r, R, \theta, p_{r}, p_{R}, p_{\theta}\right)$, as a function of the coordinates and corresponding momenta involved in the Hamilton equations which govern the classical evolution of the system. It should be noted that the PS distribution in its more general form of Eq. (5) is the modulus of the quantum PS state, and as such it has the dimensions of a PS wave function, not of a probability distribution.

The PS function of Eq. (6) and the PS state $\Phi(r, R, \theta) \tilde{\Phi}\left(p_{r}, p_{R}, j\right)$ are similar functions. In fact, the two functions coincide in coordinate space, and differ in momentum space. Actually, both the PS state and $\Phi(r, R, \theta)\left|\tilde{\Phi}\left(p_{r}, p_{R}, j\right)\right|$ are the coordinate-dependent quantum initial state times a coordinate-independent (momentum-dependent) phase, which is complex in one case and real in the other case. The above is no longer true for the more general form of the PS distribution of Eq. (5) when $\Phi(r, R, \theta)$ corresponds to an excited state, since now $\Phi(r, R, \theta) \neq$ $|\Phi(r, R, \theta)|$. However, the PS function of Eq. (5), derived from the quantum PS state, exhibits properties which reflect the quantum nature of the initial state, either for ground or for excited states. In addition, $P\left(r, R, \theta, p_{r}, p_{R}, j\right)$ presents properties which make this function a suitable one in order to sample initial conditions for classical trajectories.

Among the first type of properties there are two important ones. One is that $P\left(r, R, \theta, p_{r}, p_{R}, j\right)$ gives the correct quantum probability distribution of the initial state, both in coordinate and in momentum space, $|\Phi(r, R, \theta)|^{2}$ and $\left|\tilde{\Phi}\left(p_{r}, p_{R}, j\right)\right|^{2}$, respectively. The other property is that the average total energy associated with $P\left(r, R, \theta, p_{r}, p_{R}, j\right)$ is the same as that of the quantum initial state. This can be easily checked by averaging the quantum Hamiltonian of Eq. (1) over the PS state, $<\Phi(r, R, \theta) \tilde{\Phi}\left(p_{r}, p_{R}, j\right)|\hat{H}| \Phi(r, R, \theta) \tilde{\Phi}\left(p_{r}, p_{R}, j\right)>$, and taking into account that $\hat{H}$ depends solely upon the coordinates and derivatives of the coordinates, 
but not upon the momenta. Alternatively, the same result is obtained for the average value of the corresponding classical Hamiltonian $H$ over $P\left(r, R, \theta, p_{r}, p_{R}, j\right)$, $<P\left(r, R, \theta, p_{r}, p_{R}, j\right)|H| P\left(r, R, \theta, p_{r}, p_{R}, j\right)>$. Among the second type of properties is the fact that $P\left(r, R, \theta, p_{r}, p_{R}, j\right)$ is real and positive everywhere in phase space. All the above properties are also found in the previously proposed PS distribution $[27]$

$$
P^{\prime}\left(r, R, \theta, p_{r}, p_{R}, j\right)=|\Phi(r, R, \theta)|^{2}\left|\tilde{\Phi}\left(p_{r}, p_{R}, j\right)\right|^{2},
$$

and in the Wigner distribution. However, as discussed earlier [27], the computational cost of obtaining the distributions of Eqs. (5) and (7) (which is similar in both cases) is much lower than that of the Wigner distribution.

In the previous study [27] two slightly different PS distributions were applied. One distribution was that of Eq. (7), and the other distribution was $P^{\prime}\left(r, R, \theta, p_{r}, p_{R}, j\right) \sin \theta$, i.e. including the coordinate-dependent part of the volume element, $d v=\sin \theta d r d R d \theta d p_{r} d p_{R}$. The two initial distributions present the same shape in all the degrees of freedom, except in the angular one. It was found that $P^{\prime}\left(r, R, \theta, p_{r}, p_{R}, j\right) \sin \theta$ led to a better agreement with the quantum results that $P^{\prime}\left(r, R, \theta, p_{r}, p_{R}, j\right)$. The reason is that by including the $\sin \theta$ factor, the initial PS distribution propagated classically becomes closer in shape to the initial wave packet which is propagated quantum mechanically [27]. In this sense, $P\left(r, R, \theta, p_{r}, p_{R}, j\right)$ is even closer in shape to the quantum initial state than $P^{\prime}\left(r, R, \theta, p_{r}, p_{R}, j\right) \sin \theta$. In fact, $P\left(r, R, \theta, p_{r}, p_{R}, j\right)$ is the simplest real and positive PS function with the closest shape to the quantum initial state.

\subsection{Sampling of the initial conditions}

Once the PS distribution of Eq. (5) is generated, it can be used to sample a set of initial conditions of the system. A basic requirement of the sampling procedure used is to cause as less distortion as possible in the shape of the distribution of initial conditions sampled with respect to the original PS distribution. The sampling method used is based on a grid representation of the PS distribution. The 
$P\left(r, R, \theta, p_{r}, p_{R}, j\right)$ distribution is represented on a 6-dimensional PS grid with an associated volume element $d v$. Then a new, more sparse grid is defined by dividing the above grid into 6-dimensional boxes or cells, such that each cell, associated with a volume element $d V_{i}$, contains more than one volume element $d v$ of the original grid. Now the average value

$$
<D>{ }_{i}=\frac{1}{N} \sum_{n=1}^{N} P_{n}^{(i)}\left(r, R, \theta, p_{r}, p_{R}, j\right),
$$

is associated with each volume element $d V_{i}$, where $P_{n}^{(i)}\left(r, R, \theta, p_{r}, p_{R}, j\right)$ is the PS distribution of Eq. (5), and the summation is carried out over the values of the distribution at the $N$ 6-dimensional PS points of the denser grid contained in $d V_{i}$. If $M$ is the total number of initial conditions to be generated, $M_{i}=M\langle D\rangle_{i}$ gives the number of initial conditions to be uniformly distributed in the PS volume element $d V_{i}$.

\section{Results and discussion}

\subsection{Distributions of initial conditions}

The method described above was applied to sample initial conditions according to the PS distribution of Eq. (5). The distributions of initial conditions generated are displayed in Figs. 1 and 2 for all the coordinate and momentum degrees of freedom, and compared with the original PS distribution. In the grid sampling method the 6 -dimensional PS grid was composed of $8 \times 18 \times 43 \times 7 \times 9 \times 5$ cells in $r, R, \theta, p_{r}, p_{R}$, and $j$, respectively, in the ranges 1.9a.u. $\leq r \leq 3.2 a . u ., 6.5 a . u . \leq R \leq 9.3 a . u$., $0 \leq \theta \leq \pi,-13.9 a . u . \leq p_{r} \leq 13.9 a . u .,-9.6 a . u . \leq p_{R} \leq 9.6 a . u$. , and $0 \leq j \leq 4$. The number of initial conditions sampled was 150053 . We note that the one-dimensional distributions shown in Figs. 1 and 2 are the modulus (not the square modulus) of the corresponding function, calculated as

$$
P(r)=\left\{\int d R \int d \theta \sin \theta \int d p_{r} \int d p_{R} \sum_{j}\left[F\left(r, R, \theta, p_{r}, p_{R}, j\right)\right]^{2}\right\}^{1 / 2},
$$


and similarly for the other degrees of freedom, being $F\left(r, R, \theta, p_{r}, p_{R}, j\right)$ the PS distribution of Eq. (5) or the distribution of initial conditions.

As seen from Figs. 1 and 2, the distribution of initial conditions sampled reproduces accurately the shape of the original PS distribution for all the degrees of freedom. Despite the large number of initial conditions sampled and that we are dealing with six coupled degrees of freedom, the sampling method is found to be very efficient.

\subsection{Classical dynamics simulations}

Starting from the initial conditions previously sampled, classical trajectory calculations were carried out to simulate the ultraviolet photolysis of $\mathrm{Ar}-\mathrm{HCl}$. The trajectories were propagated up to $80 \mathrm{fs}$. At this final time, one-dimensional probability distributions associated with all the degrees of freedom were calculated, and compared with those obtained from an exact wave packet calculation. They are shown in Figs. 3 and 4. Details of the wave packet calculation have been given elsewhere [29]. It is stressed that now the distributions of Figs. 3 and 4 are true probability distributions (a square modulus), which can be compared with those obtained previously [27] using a different PS distribution.

Prior to analyzing the final classical distributions, it is illustrating to comment briefly the main features of the $\mathrm{Ar}-\mathrm{HCl}$ photodissociation dynamics [28,29,31,32]. Ultraviolet photoexcitation of the cluster to the repulsive $A^{1} \Pi$ electronic state of $\mathrm{HCl}$ deposits a large amount of energy (several $\mathrm{eV}$ ) in the $\mathrm{HCl}$ bond. The photolysis process has been found to occur dominantly through direct or nearly-direct dissociation of the hydrogen fragment. Those wave packet components associated with this dissociation mechanism give rise to the main peaks of the distributions of Figs. 3 and 4 . Some of the wave packet components populate excited-state resonances of the cluster, where the hydrogen is initially trapped in between the Ar and $\mathrm{Cl}$ atoms. Hydrogen dissociation follows in this case an indirect mechanism, through which the hydrogen collides once or more with the heavier atoms, and transfers to 
them part of its initial excess energy. This indirect photodissociation mechanism manifests itself in the lower probability tails of the quantum distributions shown in Figs. 3 and 4 . Direct dissociation of the $\mathrm{H}$ fragment is expected to be mainly a classical process. By contrast, the mechanism of indirect dissociation is subject to stronger quantum effects, such as resonances and interference effects.

By using the $P^{\prime}\left(r, R, \theta, p_{r}, p_{R}, j\right) \sin \theta$ distribution good agreement was found between the classical and the quantum results for the main peaks of the final probability distributions [27]. However, the lower probability tails of the distributions were poorly reproduced, being in general the classical tails shorter than the quantum ones (the $j$ distribution was an exception). This result was attributed to a combination of the quantum effects involved in the dynamics leading to these tails, and the influence of the $\sin \theta$ factor in the shape of the initial PS distribution. Calculations using the initial $P^{\prime}\left(r, R, \theta, p_{r}, p_{R}, j\right)$ distribution without the $\sin \theta$ factor led to somewhat more extended classical tails. However, the agreement with the quantum distributions was globally much worse in this case, while the improvement in reproducing the tails was rather small. It was concluded that quantum effects were the main responsible of the poor classical description of the distribution tails.

The main peaks of the classical distributions of Figs. 3 and 4 reproduce the quantum result similarly well as when the $P^{\prime}\left(r, R, \theta, p_{r}, p_{R}, j\right) \sin \theta$ distribution was used. However, the present classical distributions display tails more extended, in some cases (the $r, R$, and $j$ final distributions) covering almost the same range as the quantum tails. This indicates that, although the tails are still only qualitatively reproduced, the PS distribution used here is more suitable to describe the indirect dissociation mechanism. Thus, the classical results obtained with the present initial PS distribution improve over the previous results obtained with $P^{\prime}\left(r, R, \theta, p_{r}, p_{R}, j\right) \sin \theta$, since population is predicted in regions of the quantum tails where the previous distribution failed. Such an improvement is even more pronounced when the classical distributions of Figs. 3 and 4 are compared with the previous results obtained using the $P^{\prime}\left(r, R, \theta, p_{r}, p_{R}, j\right)$ distribution (without the $\sin \theta$ factor). 
Most of the discrepancies in the shape of the classical and quantum distributions are originated from quantum dynamical effects, mainly interference effects, involved in the photodissociation process. A clear example is the absence of intensity in the classical angular distributions in the region $\theta<40^{\circ}$, and the low intensity in the region $\theta>150^{\circ}$. The classical intensity found at $\theta>150^{\circ}$ corresponds to initial population in this region, from which the hydrogen dissociates directly. The above two angular regions are sterically blocked by the $\mathrm{Ar}$ and $\mathrm{Cl}$ atoms, and cannot be accessed by the $\mathrm{H}$ fragment in a classical dynamics. Quantum mechanically those angles are populated through interference between the angular components of the wave packet [29], upon the collisions of the hydrogen with Ar and Cl. The result is that the intensity which should appear at these angles in the classical distributions is transferred to other angular regions, and therefore to other regions of phase space. This causes the main differences in the shape of the classical and quantum final distributions, not only in the angular coordinate but in all the degrees of freedom. For processes less subject to quantum effects, particularly interference, than the present one, the initial PS distribution proposed in this work could lead to a more quantitative correspondence between classical and quantum predictions.

The computational cost of obtaining the new and the previous PS distributions, and generating the corresponding distributions of initial conditions is practically identical. It should be commented, however, that the calculation of final probability distributions is somewhat more costly in the present case. The difference is originated in that with the new and the previous PS distributions what is propagated is the modulus and the square modulus of a wave function, respectively. In order to calculate a final probability distribution, the modulus at final time has to be squared, and this squaring operation involves an additional cost.

\section{CONCLUSIONS}

A new quantum-type phase-space distribution to sample initial conditions for classical trajectories is proposed in this work. The first step is the definition of a 
quantum phase-space state of the system as the direct product of the coordinate and momentum representations of the quantum initial state. Then the phase-space distribution function is obtained as the modulus of such a phase-space state. Among the properties of this distribution are: $(i)$ It is real and positive everywhere in phase space; (ii) It gives the correct quantum probability distributions both in coordinate and in momentum space; (iii) It matches the correct total energy of the quantum initial state. In addition to the above properties, the present phase-space distribution function is obtained more efficiently than other distributions like the Wigner one, and resembles more closely the shape of the quantum initial state.

The suggested distribution has been applied to sample initial conditions which mimic the initial state prepared by ultraviolet excitation of the $\mathrm{Ar}-\mathrm{HCl}$ cluster. The subsequent photodissociation dynamics is simulated by means of classical trajectories. The results are compared with those of a quantum wave packet calculation, and with an earlier classical simulation. The present phase-space distribution is found to improve the agreement between the classical and the quantum final probability distributions calculated, with respect to the results obtained with the previously suggested distribution. Specifically, with the current initial distribution the final probability distributions are able to predict intensity in regions where the previous classical simulation failed. Such an improvement is attributed to the fact that the initial distribution proposed here resembles more closely the shape of the initial state propagated quantum mechanically. The discrepancies still remaining between the classical and quantum predictions are attributed, to a large extent, to quantum interference effects involved in the process studied, and not taken into account in the classical simulation. We envision application of the proposed PS distribution to problems where all the modes are described classically, and in hybrid quantumclassical methods [9(b),33,34], where some of the modes are described quantum mechanically, and the remaining modes are treated classically. 


\section{ACKNOWLEDGMENTS}

This work was supported by C.I.C.Y.T. (Ministerio de Ciencia y Tecnología), Spain, Grant No. BFM-2001-2179, and by the European Network TMR, Grant No. HPRN-CT-1999-00005.

\section{References}

[1] H.-W. Lee, M.O. Scully, J. Chem. Phys. 73 (1980) 2238.

[2] J.C. Gray, D.G. Truhlar, J. Chem. Phys. 76 (1982) 5350.

[3] R. Bershon, M. Shapiro, J. Chem. Phys. 85 (1986) 1396.

[4] H. Guo, J.N. Murrell, Mol. Phys. 65 (1988) 821.

[5] V. Engel, R. Schinke, J. Chem. Phys. 88 (1988) 6831.

[6] A. García-Vela, P. Villarreal, G. Delgado-Barrio, J. Chem. Phys. 92 (1990) 6504; ibid. 94 (1991) 7868.

[7] R. Alimi, R.B. Gerber, V.A. Apkarian, Phys. Rev. Lett. 66 (1991) 1295.

[8] Y. Yan, R.M. Whitnell, K.R. Wilson, A.H. Zewail, Chem. Phys. Lett., 193 (1992) 402.

[9] (a) A. García-Vela, R.B. Gerber, J.J. Valentini, Chem. Phys. Lett. 186 (1991) 223; (b) J. Chem. Phys. 97 (1992) 3297.

[10] E.D. Potter, Q. Liu, A.H. Zewail, Chem. Phys. Lett. 200 (1992) 605.

[11] G. Lendvay, G.C. Schatz, J. Chem. Phys. 96 (1992) 4356; J. Phys. Chem. 96 (1992) 3752.

[12] Z. Li, A. Borrmann, C.C. Martens, J. Chem. Phys. 97 (1992) 7234; X. Hu and C.C. Martens, ibid. 97 (1992) 8805. 
[13] L.M. Finney, C.C. Martens, J. Phys. Chem. 97 (1993) 13477.

[14] A.B. McCoy, Y. Hurwitz, R.B. Gerber, J. Phys. Chem. 97 (1993) 12516.

[15] B. Schmidt, R.B. Gerber, Phys. Rev. Lett. 72 (1994) 2490.

[16] A.I. Krylov, R.B. Gerber, J. Chem. Phys. 106 (1997) 6574.

[17] S. Fernández Alberti, N. Halberstadt, J.A. Beswick, J. Echave, J. Chem. Phys. 109 (1998) 2844.

[18] S. Fernández Alberti, N. Halberstadt, J.A. Beswick, A. Bastida, J. Zúñiga, A. Requena, J. Chem. Phys. 111 (1999) 239; A. Bastida, B. Miguel, J. Zúñiga, A. Requena, N. Halberstadt, K.C. Janda, ibid. 111 (1999) 4577.

[19] M.Y. Niv, A.I. Krylov, R.B. Gerber, U. Buck, J. Chem. Phys. 110 (1999) 11047.

[20] E. Wigner, Phys. Rev. 40 (1932) 749.

[21] J.G. Kirkwood, Phys. Rev. 44 (1933) 31.

[22] K. Husimi, Proc. Phys. Math. Soc. Jpn. 22 (1940) 264; K. Takahashi, Prog. Theor. Phys. Suppl. 98 (1989) 109.

[23] H.W. Lee, Phys. Rep. 259 (1995) 147.

[24] K.M. Christoffel, J.M. Bowman, J. Chem. Phys. 104 (1996) 8348.

[25] A. García-Vela, J. Rubayo-Soneira, G. Delgado-Barrio, P. Villarreal, J. Chem. Phys. 104 (1996) 8405; G. Delgado-Barrio, A. García-Vela, C. García-Rizo, M.I. Hernández, P. Villarreal, Mol. Eng. 7 (1997) 219.

[26] B. Segev, Phys. Rev. A 63 (2001) 52114.

[27] A. García-Vela, J. Chem. Phys. 112 (2000) 8302.

[28] A. García-Vela, R.B. Gerber, J. Chem. Phys. 103 (1995) 3463. 
[29] A. García-Vela, J. Chem. Phys. 108 (1998) 5755.

[30] J.A. Beswick, J. Jortner, Adv. Chem. Phys. 47 (1981) 363.

[31] J.C. Juanes-Marcos, A. García-Vela, J. Chem. Phys. 111 (1999) 2606; ibid. 112 (2000) 4983; ibid. 115 (2001) 5692 (E).

[32] J.C. Juanes-Marcos, A. García-Vela, J. Phys. Chem. A 106 (2002) 236.

[33] L. Liu, H. Guo, J. Chem. Phys. 104 (1996) 528.

[34] J.-Y. Fang, C.C. Martens, J. Chem. Phys. 104 (1996) 3684. 


\section{FIGURE CAPTIONS}

Fig. 1. One-dimensional distributions in the coordinates, corresponding to the initial state of the $\mathrm{Ar}-\mathrm{HCl}$ cluster in phase space. The solid curves are obtained from the phase-space distribution defined in Eq. (5), while the histograms correspond to the distribution of initial conditions generated with the grid sampling method described in the text.

Fig. 2. Same as Fig. 1 but for the momenta.

Fig. 3. One-dimensional probability density distributions in the coordinates at the end of the dynamical simulation. The solid curves and the histograms correspond to the quantum and classical distributions, respectively. The factor $\sin \theta$ has been included in the $\theta$ distribution.

Fig. 4. Same as Fig. 3 but for the momenta. 